\title{
Comparison between Watermelon Frost and Ribavirin Aerosol in the Treatment of Oral Herpes in Hand, Foot and Mouth Disease
}

\author{
Jingzhi Zhong ${ }^{1}$, Yueyan Huang ${ }^{2 *}$, Qingfeng Li $^{2}$, Suren R Sooranna ${ }^{3 *}$, Yunfeng Huang ${ }^{2}$, Zhijing \\ Huang ${ }^{2}$, Shuangshuang Lu², Hongbo Gao' ${ }^{2}$ Linyang Yuan $^{2}$, Anna Nong ${ }^{2}$, Lianghua Liao² and \\ Zhenyi Cen ${ }^{2}$
}

${ }^{1}$ Department of Pediatrics, First Affiliated Hospital of Guangxi Medical University, NanNing, China

${ }^{2}$ Department of Pediatrics, Affiliated Hospital of YouJiang Medical College for Nationalities, BaiSe, China

${ }^{3}$ Department of Surgery and Cancer, Imperial College London, Chelsea and Westminster Hospital, 369, Fulham Road, London SW10 9NH, $U K$

*Corresponding author: Yueyan Huang, Department of Pediatrics, Affiliated Hospital of YouJiang Medical University for Nationalities, Bai Se, GuangXi, China

Suren R Sooranna, Department of Surgery and Cancer, Imperial College London, Chelsea and Westminster Hospital, 369, Fulham Road, London

\section{ARTICLE INFO}

Received: February 14, 2020

Published: March 02, 2020

Citation: Jingzhi Z, Yueyan H, Qingfeng L, Suren R S, Yunfeng H, et al., Comparison between Watermelon Frost and Ribavirin Aerosol in the Treatment of Oral Herpes in Hand, Foot and Mouth Disease. Biomed J Sci \& Tech Res 26(2)-2020. BJSTR. MS.ID.004314.

Keywords: Watermelon Frost; Ribavirin Aerosol; Oral Herpes; Foot and Mouth Disease

\section{ABSTRACT}

Objectives: We evaluated the effect and safety of using ribavirin aerosol and watermelon frost therapy in children with mild hand, foot, and mouth disease (HFMD) by conducting a prospective and randomized trial.

Methods: 369 mild HFMD patients were randomly assigned to being treated with either ribavirin or watermelon frost. The course of treatment was 5 days. After this period, the effect of the treatment was evaluated, including the time of disappearance of oral ulcers, fever clearance and vesicles in the hand or foot, together with the clinical grading of the symptoms.

Results: 353 patients completed the trial. Watermelon frost reduced the time to the first disappearance of oral ulcers and vesicles from hand or foot in 4.3 days (95\% CI, 3.9 to 4.6 days), compared with 5.5 days (95\% CI, 4.9 to 6.0 days) in the patients treated with ribavirin $(\mathrm{p}<0.0036)$. There was no significant difference between the two treatment groups with respect to coughing and fever. No adverse reaction was found in either of the two groups.

Conclusion: Watermelon frost was better than ribavirin Aerosol for the treatment of oral herpes in HFMD with no adverse reactions. Owing to its convenience and cost effectiveness, our data suggest that watermelon frost could replace ribavirin in the treatment of oral herpes in mild HFMD.

\section{Introduction}

Hand, Foot and Mouth Disease (HFMD) is a common infectious disease in children with the characteristics of rapid transmission and strong infection. More than $70 \%$ cases are caused by enterovirus 71 (EV71), Coxsackie virus type A 16 (CVA16) and other enterovirus infections [1,2]. The clinical manifestations of HFMD typically begin with a fever, sore throat, headache, tiredness and anorexia. One to two days later, blisters appear in the mouth and rashes develop on the cheeks, gums and tongue. In recent years, HFMD has become more and more common in the Asia Pacific region, especially in China [3]. In 2008, hand, foot and mouth disease were declared as the legal C class infectious disease in China. A survey by the Ministry of Health of China in 2008-2012 demonstrated that the estimated 
incidence of HFMD is 1.2 per 1,000 persons per year in China and the disease is responsible for 350-900 reported deaths annually [4]. HFMD is usually self-limiting with most sufferers manifesting a mild form of the disease, with relief of symptoms seen within 7-10 days [5].

However, a few cases can progress into a severe condition with complications, including encephalitis, neurogenic pulmonary edema, pulmonary hemorrhage and circulatory failure. These complications are life threatening if treatment is not given promptly [6]. Oral herpes is one of common symptoms of mild HFMD. This normally causes pain of oral ulcers which interfere with the diet and can even lead to dehydration and liquid imbalance. Currently, there are no specific treatments for HFMD. The most common treatment is only to relieve the offending symptoms by supportive therapy and the use of antiviral agents and immune-potentiators. In this study, we conducted a clinical trial to study the efficacy and safety of both ribavirin aerosol and watermelon frost in treating oral herpes brought about by mild HFMD cases.

\section{Patients and Methods}

\section{Study Design}

Eligible participants were recruited from March 2016 to March 2018 from the Outpatient Departments of Pediatrics in the Affiliated Hospital of YouJiang Medical University for Nationalities and the first Affiliated Hospital of Guangxi Medical University. This study was performed with the approval of the respective Ethics Committees of the both hospitals involved.

\section{Inclusion Criteria}

All patients were younger than 6 years old and were clinically diagnosed with HFMD according to the 'Guidelines for HFMD Diagnosis and Treatment' issued by the Ministry of Health of China (version 2010), published by the National Health and Family Planning Commission. Up to 72 hours of the initial diagnosis, patients were not subjected to any antiviral agents or analgesicantipyretics. The clinical symptoms ruled out any other diseases.

\section{Exclusion Criteria}

Severe HFMD cases were excluded from the study as well as cases which appeared to be developing into severe HFMD cases during the period of the study. Patients who had taken antivirals (including Chinese patent medicines) 7 days before diagnosed. Cases complicated with bacterial infections (C-reactive protein $>8 \mathrm{mg} / \mathrm{L}$ ) and participants who were allergic to ribavirin or watermelon as well as patients who had participated in other clinical studies within the last 30 days were also excluded.

\section{Randomization}

All participants were randomly grouped into watermelon or ribavirin groups. There were 187 cases in the treatment group, who received watermelon frost and the ribavirin groupwith182 cases received ribavirin aerosol. According to the group, participants were registered and provided with the drugs which were used immediately.

\section{Methods}

Both groups followed the "Diagnosis and treatment guidelines of HFMD (2010 edition)" in order to follow the supportive therapy. The ribavirin group used ribavirin aerosol (Shanghai Sine Pharmaceutical Company Limited). This drug should first be taken 4 times in the first hour, followed by once every other hour; after 2 days, it should be taken 4 times every day. Each time it is taken, the dose applied should be that the aerosol be pressed 2-3 times. The average amount of drug received by the recipient would be approximately $15-20 \mathrm{mg}$ (this is equivalent to $30-40$ presses of the aerosol with every press releasing $0.5 \mathrm{mg}$ drug). The treatment should be continued for a period of 7 days. The watermelon frost group used the Chinese medicine watermelon frost (produced by Guilin Sanjin Pharmaceutical Company Limited) for 7 days. The regimen for this drug was simpler with 3 applications per day. Each application consisted of 2-3 presses of the applicator. If necessary, watermelon frost could be applied directly to where the oral ulcer was located.

\section{Evaluating Indexes}

We followed up the patients in the two groups every day in the treatment period. The concrescence time of oral ulcer before and after the treatment was recorded and the clinical symptoms were scored. With both groups of patients, the disappearance time of fever and roseola from the hands and feet were observed and recorded, and the clinical curative effect as well as the comprehensive curative effect were evaluated.

\section{Curative Effect Standards}

\section{Oral Herpes:}

1) Recovery: oral herpes disappeared, receiving the correct diet and no obvious drooling noted;

2) Excellent: the amount of herpes reduced to more than half of the primary and an obvious improvement in the diet;

3) Effective: the amount of herpes receded to approximately $1 / 3-1 / 2$ of the initial amount seen and a slight improvement of the diet;

4) Invalid: the amount of herpes seen was the same or was increased compared to the initial amount seen and the diet was not better.

\section{Fever Symptoms:}

1) Recovery: the temperature reverted to normal (anal temperature $\leq 37.5^{\circ} \mathrm{C}$ );

2) Excellent: the base temperature decreased by $\geq 1^{\circ} \mathrm{C}$;

3) Effective: the base temperature decreased by $0.5 \sim 0.9^{\circ} \mathrm{C}$; 
4) Invalid: the temperature decreased $\leq 0.5^{\circ} \mathrm{Cor}$ was increased.

The clinical symptoms, including hand and foot rash and coughing:

1) Recovery: the clinical symptoms score was zero;

2) Excellent: the score was reduced $\geq 2$;

3) Effective: the score was reduced $\geq 1$ and $<2$;

4) Invalid: the score had not changed or was increased (the curative effect evaluation of each single clinical symptom was directed only to the single clinical symptom deviant).

\section{Safety Assessment}

The safety assessment index included any adverse events, laboratory examination and any changes of lifestyle during seen from the pre-therapy to the post-treatment period observed during the clinical treatment.

\section{Statistical Analysis}

All statistical procedures were performed with SPSS16.0 software. The enumeration data were expressed as percentages and the measurement data were expressed as the means \pm SD $(\chi \pm s)$. All reported $P$ values were two-sided, and all $P$ values were statistically significant if $\mathrm{P}<0.05$. The category variables were compared by the chi-squared test and the continuous variables of two groups were compared by group t-test.

\section{Results}

380 cases met the inclusion criteria. Of the380 participants, 4 participants were excluded because they were treated with antiviral medicines within 7 days before the administration. 2 were excluded because they were allergic to ribavirin or watermelon frost. 5 were excluded because their CRP $>8 \mathrm{mg} / \mathrm{L}$. 369 cases were enrolled in our study. 178 cases from the watermelon frost group completed the trial. 5 cases were excluded because they developed into severe HFMD and another 4 cases were lost during the trial. 175 cases from the ribavirin group completed the trial in which5 cases were lost and two cases developed for severe HFMD (Fig. 1). There was no statistically significant difference between of lost patients in the two groups. The baseline demographic characteristics, clinical features, and laboratory variables were similar between the two groups of patients (Table 1).

\section{Curative Effect of Oral Herpes}

After 5 days of administration, the effect on the oral ulcers of the watermelon frost group was superior to the ribavirin group $(\chi 2=4.338, \mathrm{P}=0.037 ;$ Table 2$)$.

\section{Fever Clearance}

After 5 days, the grade of fever was $0.05 \pm 0.29$ both in the watermelon and in the ribavirin groups and there was no statistical difference between the groups $(\mathrm{z}=0.18, \mathrm{P}=0.8589)$. The temperature of patients in the watermelon frost group dropped to lower than the ribavirin group ( $\chi 2=6.776, \mathrm{P}=0.009$; Table 2).

\section{Clinical Outcomes}

5 days after the initial treatment, the difference of the development of skin rash was not statistically significant $(\chi 2=0.081$, $\mathrm{P}=0.776$; Table 2). The score for coughing was $0.07 \pm 0.25$ in the watermelon frost group and $0.17 \pm 0.42$ in the ribavirin group before using the drugs. After 5 days of treatment, the scores in both the watermelon and ribavirin groups were $0.03 \pm 0.18$ and therefore there was no statistical difference between the two groups $(\chi 2=3.202, \mathrm{P}=0.074$; Table 2$)$. The scores for fever were $0.93 \pm 0.96$ and $1.03 \pm 1.04$ in the treatment and ribavirin groups, respectively, before using the drugs. After 5 days, the scores were reduced to $0.05 \pm 0.29$ both groups $(\mathrm{z}=0.18, \mathrm{P}=0.8589)$ (Table 2 \& Figure 1$)$.

Table 1: Characteristics of the participants with HFMD.

\begin{tabular}{|c|c|c|c|}
\hline Characteristic & Watermelon frost group & Ribavirin group & P \\
\hline Male, $\mathrm{n}(\%)$ & $82(46.1)$ & $88(50.3)$ & 0.428 \\
\hline Female, $\mathrm{n}(\%)$ & $96(53.9)$ & $26.99 \pm 8.41$ & 0.629 \\
\hline Mean age \pm SD, (M) & $26.63 \pm 9.06$ & $39.16 \pm 0.26$ & 1.489 \\
\hline Mean Temperature \pm SD, ${ }^{\circ} \mathrm{C}$ & $39.20 \pm 0.28$ & $2.86 \pm 0.34$ & - \\
\hline Oral herpes & $2.90 \pm 0.25$ & - & - \\
\hline Symptom & - & $2.49 \pm 0.45$ & - \\
\hline Cough & $2.61 \pm 0.51$ & $2.68 \pm 0.47$ & - \\
\hline
\end{tabular}

Note: Oral herpes score: 0: no herpes in isthmus of fauces; 1 : Herpes distributed within 1/2 isthmus of fauces; 2 : Herpes distributed more than 1/2 isthmus of fauces; 3 : Herpes distributed overlapping the isthmus of fauces.

Cough: 1: some coughing, no dysphoria 2: Cough frequently, no impact on the sleep quality, sometimes progressed be dysphoria, but easily to comfort; 3: Severe coughing, waking at night because of coughs, easily progress to dysphoria, difficult to comfort.

Skin rash: 1: Rash distributed in one place of the skin; 2: Rash distributed in two places of the skin; 3: Rash distributed in more than three places of the skin. 
Table 2: The outcome after 5 days of treatment with watermelon frost or ribavirin aerosol.

\begin{tabular}{|c|c|c|c|c|c|c|c|c|}
\hline Symptoms & Group & $\begin{array}{c}\text { Total } \\
\text { number }\end{array}$ & $\begin{array}{l}\text { Recovery } \\
\text { number }\end{array}$ & $\begin{array}{c}\text { Excellent } \\
\text { number }\end{array}$ & $\begin{array}{l}\text { Effective } \\
\text { number }\end{array}$ & $\begin{array}{l}\text { Invalid } \\
\text { number }\end{array}$ & $\mathrm{X} 2$ & $\mathbf{P}$ \\
\hline \multirow[t]{2}{*}{ Oral herpes } & Watermelon & 178 & 86 & 49 & 35 & 8 & 4.338 & 0.037 \\
\hline & Ribavirin & 175 & 51 & 35 & 71 & 18 & & \\
\hline \multirow[t]{2}{*}{ Fever } & Watermelon & 178 & 79 & 54 & 33 & 12 & 6.776 & 0.009 \\
\hline & Ribavirin & 175 & 58 & 40 & 50 & 27 & & \\
\hline \multirow[t]{2}{*}{ Skin rash } & Watermelon & 178 & 47 & 68 & 55 & 8 & 0.081 & 0.776 \\
\hline & Ribavirin & 175 & 43 & 62 & 61 & 9 & & \\
\hline \multirow[t]{2}{*}{ Coughing } & Watermelon & 178 & 21 & 40 & 75 & 42 & 3.202 & 0.074 \\
\hline & Ribavirin & 175 & 30 & 56 & 61 & 28 & & \\
\hline
\end{tabular}

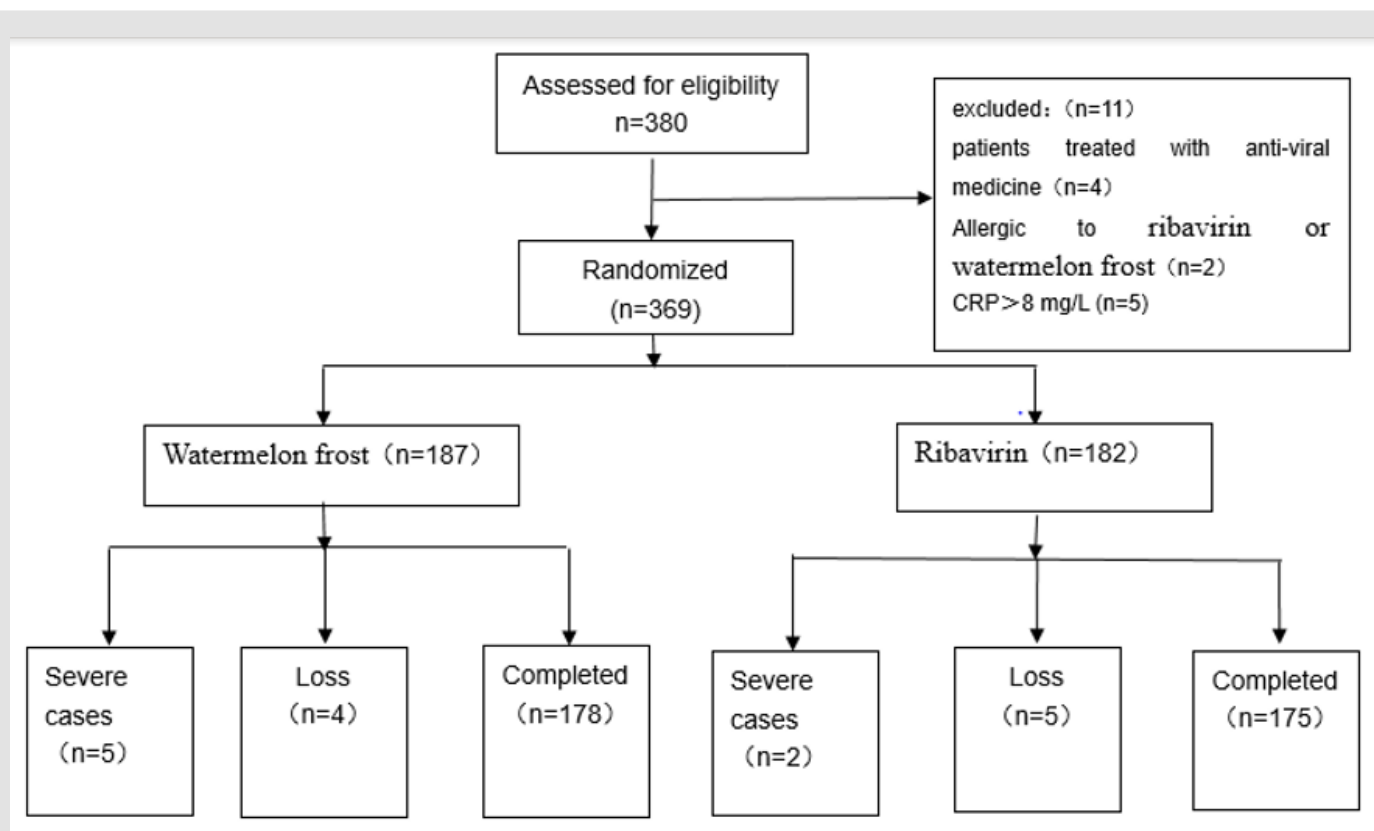

Figure 1: Flow diagram of patients in this study.

\section{Safety}

During the period of treatment, the vital signs of the two groups were without any obvious difference and were not statistically different between the two groups. There was no statistical difference between the levels of white blood cells, platelets and hemoglobin in the two groups after the treatment. The white blood cells count of 2 patients in the ribavirin group decreased to $3.5 \times 109$ $\angle \mathrm{L}$ and $4.2 \times 109 / \mathrm{L}$ in the two groups, respectively. There was no serious adverse event observed in the two groups. One case was considered to be meningitis with increasing temperature, heart rate, and breathing. These symptoms were deemed not to be related to the drugs used in this study.

\section{Discussion}

HFMD is a highly infectious disease that is spread mainly by secretions and touching. Since the first report of HFMD in 1953, this disease has caused several epidemics. However, from 2007, outbreaks and prevalence of HFMD had occurred in many regions of China, causing hundreds of child deaths every year [4]. Recent studies showed that the main reasons for HFMD epidemics are alternating infections of EV71 and CA16, the main pathogens associated with HFMD. The painful oral ulcers associated with the disease often causes difficulty in eating and even dehydration and liquid imbalances, especially in very young infants [7]. It is anticipated that controlling the development of herpes in the early periods could decrease the spread of HFMD and therefore relieve the patients' pain and improve their quality of life. According to " Diagnosis and treatment guidelines of HFMD (2010 edition)", the normal treatment includes general therapy and symptomatic treatment, and recommends the use of Chinese herbs such as antiviral oral liquids. Some studies presented suggest that only Chinese herbs or when it is combined with other antiviral drugs could efficiently improve the clinical symptoms of HFMD patients [8].

The common dosage forms of ribavirin given to patients are injections, pills, liquid, spray and aerosol. The common sideeffects of oral liquid or injections include hypo-leucocytosis, anemia, elevated serum transaminase and bilirubin, weight loss 
and growth retardation [9]. During the injections, it is possible to have the formation of red papules or the occurrence of urticaria, bronchial asthma and a transfusion reaction, which limits its application in pediatric medicine. Pharmacokinetics studies have verified that the concentration of ribavirin absorbed through the respiratory tract was 500-1000 times than those of the plasma peak concentration. The half-lifeof ribavirin in the circulation is only 2 hours and it has very few side-effects [9]. In China, ribavirin is not only used for treating Hepatitis C Virus (HCV) and Respiratory Syncytial Virus (RSV) infections, butit is also used in other antiviral treatments. Some studies showed that the rate of utilization of ribavirin in HFMD was $20 \%$. However, the regulations for the use of ribavirin is strict in many countries, including Hong Kong, is strictly regulated. Evidence-based medicine studies and random doubleblind trial results were not enough to prove that oral granules or the spray form of ribavirin could treat HFMD efficiently, or relieve the symptoms, and the leakage of aerosols could contaminate the hospital environment and increase its potential teratogenicity.

Overall, it is a matter of urgency to find an alternative safe antiviral drug to replace ribavirin in pediatric medicine. Watermelon frost consists of watermelon emulsion, forging borax, cortex phellodendri, coptis, sophorae tonkinensis, pardanthus, fritillary bulb, indigo, borneol, rheum, muhan fruit (charcoal), scutellaria, liquorice, menthol and has been shown to have numerous functions including bacteriostatic inflammation, relief of swelling and pain as well as muscular promotion. Many studies have demonstrated watermelon to have an efficient effect on stomatitis, oral ulcer and thrush $[10,11]$. Several recent clinical studies had proven that both ribavirin and watermelon frost have efficacy dealing with HFMD $[12,13]$. However, these studies had some limitations, such as these trials were restricted to a small sample and therefore cannot reflect the general population. Also, there is no trial, as yet, to compare the efficacy and safety between the two drugs in HFMD treatment. In this study, we conducted a clinical randomized controlled trial between the use of ribavirin and watermelon frost in the treatment of HFMD.

The total efficacy rate of the watermelon frost group is higher than the ribavirin group. Monitoring the vital signs and whole blood routine parameters during the treatment period, there was no statistical difference between the two groups of white blood cells, platelets and hemoglobin before and after treatment. There was no adverse reaction such as dizziness or vomiting in either group. Our study illustrated that ribavirin aerosol and watermelon frost are both effective for treating mild HFMD oral herpes. But there was no statistical difference between the two groups for the curative effect on coughing and fever associated with the disease. There was no adverse reaction in either of the two groups and both drugs performed with respect to safety. In summary, mild HFMD is a viral infection and so watermelon frost could replace ribavirin aerosol to treat oral herpes. Watermelon frost is convenient, shows no adverse reaction and avoids the side-effects associated with ribavirin. Children tend to have a good compliance with watermelon frost, and it can relieve their pain as well as the burden on medical staff. Watermelon frost has the potential of being of a good product for future use in pediatric medicine.

\section{Acknowledgement}

We are grateful to all the members which participate in this study.

\section{Funding}

The project was supported by a Self-finance Guangxi Medical Fund to J.Z. (No. Z2013047).

\section{Availability of Data and Materials}

The data supporting the findings detailed in this paper are presented in the 2 tables and 1 figure within the main paper and are available for inspection from Drs Jingzhi Zhong (842412593@ qq.com) and Suren Sooranna (s.sooranna@imperial.ac.uk).

\section{Authors' Contributions}

All the authors except YH and SRS were involved in the acquisition and analysis of the data. JZ, YH and SRS were involved with the interpretation of the data and helped to draft the manuscript. JZ and YH conceived of the study and participated in its design and coordination. All authors were involved in drafting/ revising and approving the manuscript.

\section{Ethics Approval and Consent to Participate}

This study was approved by the Ethics Committees of the Affiliated Hospital of Youjiang Medical University for Nationalities, BaiSe, PR China and the First Affiliated Hospital of Guangxi Medical University, NanNing, PR China in accordance with the Declaration of Helsinki.

\section{Patient Consent for Publication}

All participants provided written informed consent for their willingness to participate in this study.

\section{Competing Interests}

The authors declare that there is no conflict of interest.

\section{References}

1. Mao Q, Wang Y, Yao X, Bian L, Wu X, et al. (2014) Coxsackievirus A16: epidemiology, diagnosis, and vaccine. Human vaccines \& immunotherapeutics 10(2): 360-367.

2. Solomon T, Lewthwaite P, Perera D, Cardosa MJ, Mc Minn P, et al. (2010) Virology, epidemiology, pathogenesis, and control of enterovirus 71 . The Lancet Infectious diseases 10(11): 778-790.

3. Yin DQ, Wang CB, Wang CB, Xiao Z, Ji SX (2018) Epidemiology Characteristics of Human Coxsackievirus A16 and Enterovirus 71 Circulating in Linyi, China, from 2009 to 2017. Japanese journal of infectious diseases 71(6): 470-473.

4. Xing W, Liao Q Viboud C, Zhang J, Sun J, et al. (2014) Hand, foot, and mouth disease in China, 2008-12: an epidemiological study. Lancet Infect Dis 14(4): 308-318. 
5. Cox B, Levent F (2018) Hand, Foot, and Mouth Disease. Jama 320(23): 2492.

6. Mirand A, Peigue Lafeuille $H$ (2017) Clinical characteristics and course of hand, foot, and mouth disease]. Arch Pediatr 24(10): 1036-1046.

7. Ventarola D, Bordone L, Silverberg N (2015) Update on hand-foot-andmouth disease. Clin Dermatol 33(3): 340-346.

8. Li XH, Zhang X, Ding JB, Xu Y, Wei D, et al. (2014) Comparison between Chinese Herbal Medicines and Conventional Therapy in the Treatment of Severe Hand, Foot, and Mouth Disease: A Randomized Controlled Trial. Evid-Based Compl Alt.

9. Lee DU, Je SH, Yoo SJ, Kwon T, Shin JY, et al. (2017) Hematological adverse effects and pharmacokinetics of ribavirin in pigs following intramuscular administration. J Vet Pharmacol Ther 40(5): 561-568.

\section{ISSN: 2574-1241}

DOI: 10.26717/BJSTR.2020.26.004314

Yueyan Huang, Suren R Sooranna. Biomed J Sci \& Tech Res

CC (i) This work is licensed under Creative

Submission Link: https://biomedres.us/submit-manuscript.php
10. Zhao X, HeX,Zhong X (2016) Anti-inflammatory and in-vitro antibacterial activities of Traditional Chinese Medicine Formula Qingdaisan. BMC Complement Altern Med 16(1): 503.

11. Dai MR, Ho DK, Huang L, Quock RL, Flaitz CM (2016) Utilization of Chinese Herbal Medicine for Selected Oral Conditions in Two Pediatric Populations. Pediatr Dent 38(4): 311-316.

12. Lin H, Zhou J, Lin K, Wang H, Liang Z, et al. (2016) Biomed Res Int Efficacy of Scutellaria baicalensis for the Treatment of Hand, Foot, and Mouth Disease Associated with Encephalitis in Patients Infected with EV71: A Multicenter. Retrospective Analysis. Biomed Res Int.

13. Zhang HP, Wang L, Qian JH, Cai K, Chen YH, et al. (2014) Efficacy and safety of ribavirin aerosol in children with hand-foot-mouth disease. Zhongguo Dang Dai Er Ke Za Zhi 16(3): 272-276.

$\begin{array}{ll}\text { BIOMEDICAL } & \text { Assets of Publishing with us } \\ \text { RESEARCHES } & \text { - Global archiving of articles } \\ \text { - Immediate, unrestricted online access }\end{array}$

